\section{Hemoptisis recurrente asociada a falla renal y hematuria: otra de las mil caras de la enfermedad relacionada a IgG4}

\author{
DANIEL ERLIJ $^{1}$, ÁNGELA RIVERA ${ }^{1}$, JUAN CARLOS MAYA ${ }^{1}$, \\ CAROLINA CUELLAR ${ }^{1}$, GONZALO CORREA ${ }^{2}$, \\ SUSANA MICHALLAND ${ }^{1}$, GONZALO P. MÉNDEZ ${ }^{3}$
}

\section{IgG4 associated nephritis and recurrent hemoptysis. Case report}

IgG4 disease is a multi-systemic condition involving pancreas, salivary glands and lymph nodes. Less frequently, it causes interstitial nephritis and involves the lungs. We report a 58 years old male with a four years history of hemoptysis and renal dysfunction characterized by hematuria and proteinuria, responsive to steroidal therapy. The renal biopsy established the diagnosis of IgG4 associated interstitial nephritis. Lung involvement was considered secondary to the same systemic disease.

(Rev Med Chile 2017; 145: 131-134)

Key words: Hemoptysis; Immunoglobulin G; Nephritis, Interstitial.
'Sección de Reumatología, Servicio de Medicina Hospital del Salvador, Universidad de Chile. Santiago, Chile. ${ }^{2}$ Sección de Nefrología, Servicio de Medicina Hospital del Salvador. Santiago, Chile. ${ }^{3}$ Departamento de Anatomía Patológica, Facultad de Medicina, Pontificia Universidad Católica de Chile. Santiago, Chile

Fuente de apoyo financiero: ninguna.

Recibido el 30 de junio de 2016, aceptado el 21 de octubre de 2016

Correspondencia a:

Dr. Daniel Erlij

Sección de Reumatología,

Servicio de Medicina Hospital del Salvador.

Avenida Salvador 364,

Providencia, Santiago

Teléfono: 25753590

danerlij@gmail.com
L a enfermedad relacionada a IgG4 (ER-IgG4), es una entidad fibroinflamatoria sistémica que puede afectar a múltiples órganos, considerándose un "gran simulador" de la medicina. Si bien el compromiso más reconocido es el pancreático, está descrita la afectación de vía biliar, pericardio, linfonodos, pulmón, retroperitoneo y riñón, manifestándose como pseudotumores, organomegalia y derrame, lo cual obliga a plantear como diagnóstico diferencial un gran número de patologías ${ }^{1}$.

\section{Caso clínico}

Varón de 58 años, tiene antecedentes de gota e hipertensión arterial, es usuario de marcapaso desde el 2011 por ritmo nodal y es monorreno quirúrgico por donación de riñón derecho hace más de treinta años a hermana con enfermedad renal de etiología desconocida. Consulta el 2011 por cuadro de 3 meses de compromiso del estado general, asociado a episodios de hemoptisis de baja cuantía, a lo cual se suma 2 semanas previo a su evaluación, monoartritis de tobillo izquierdo, la cual se automedica con colchicina y alopurinol.
Posterior a ello aparecen lesiones purpúricas en extremidades inferiores. Destaca en exámenes una eosinofilia de $3.300 \mathrm{x} \mathrm{mm}^{3}$, VHS $90 \mathrm{~mm} / \mathrm{h}$, ascenso de su creatinina basal de 1,5 a 3,69 mg/dL con hematuria no dismórfica de $50 \mathrm{x}$ campo y proteinuria (+). Se tomó PPD que fue de $18 \mathrm{~mm}$, serología viral (VIH, hepatitis B y C), negativas. Anticuerpos antinucleares (ANA) (-), anticitoplasma de neutrófilos (ANCA) (+) leve de patrón indeterminado, con ELISA para PR3 y MPO (-), antimembrana basal (-), antiestreptolisina (-), complemento C3: $34 \mathrm{mg} /$ dL (normal 90-180), C4: 1,0 mg/dL (normal 1040). CT de tórax y abdomen: nodulillo pulmonar de aspecto benigno en base derecha, además de una zona de vidrio esmerilado y bronquiectasias en ambos campos. A nivel abdominal se observó nefromegalia (Figura 1). Fibrobroncoscopía con lavado bonquioalveolar descarta infección, con baciloscopías (-). Se interpreta como cuadro vasculítico y se indica metilprednisolona $500 \mathrm{mg} /$ día durante 3 días, recuperando creatinina hasta $1,49 \mathrm{mg} / \mathrm{dL}$. Es dado de alta con prednisona y colchicina. El paciente descontinúa tratamiento y consulta al año siguiente por mismo cuadro de hemoptisis y falla renal asociado a hematuria y 


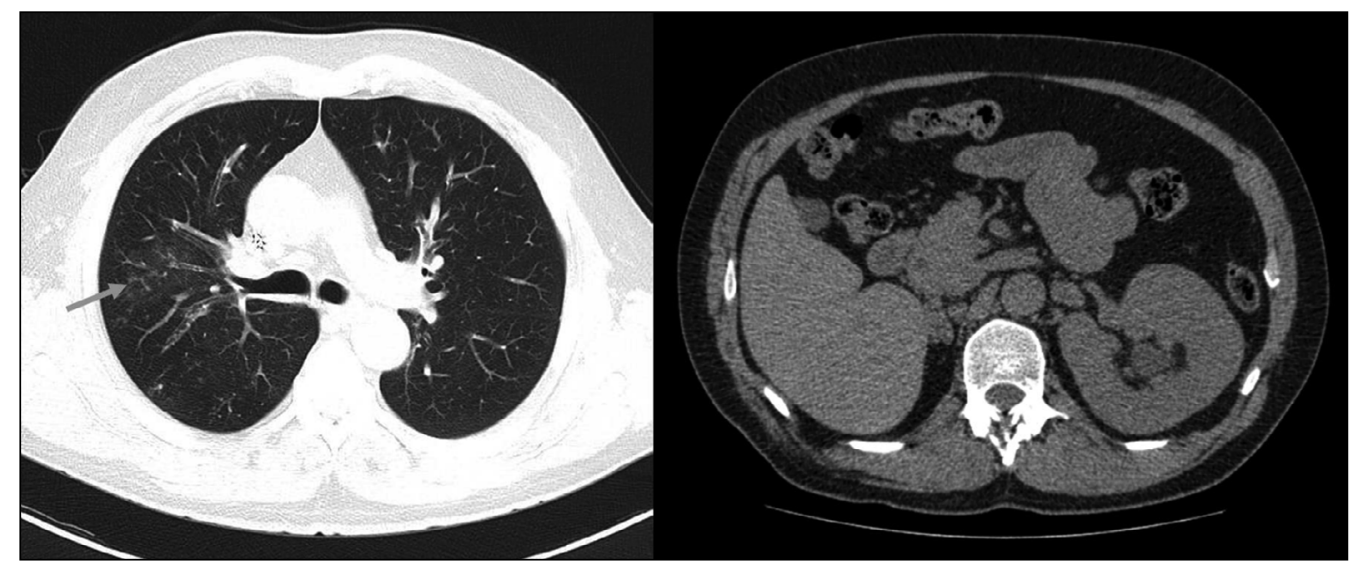

Figura 1. Tomografía axial computada (TAC) sin contraste. Izquierda: corte de tórax en ventana pulmonar que permite observar zona de vidrio esmerilado (flecha). Derecha: corte de abdomen que evidencia presencia de nefromegalia izquierda (paciente monorreno).

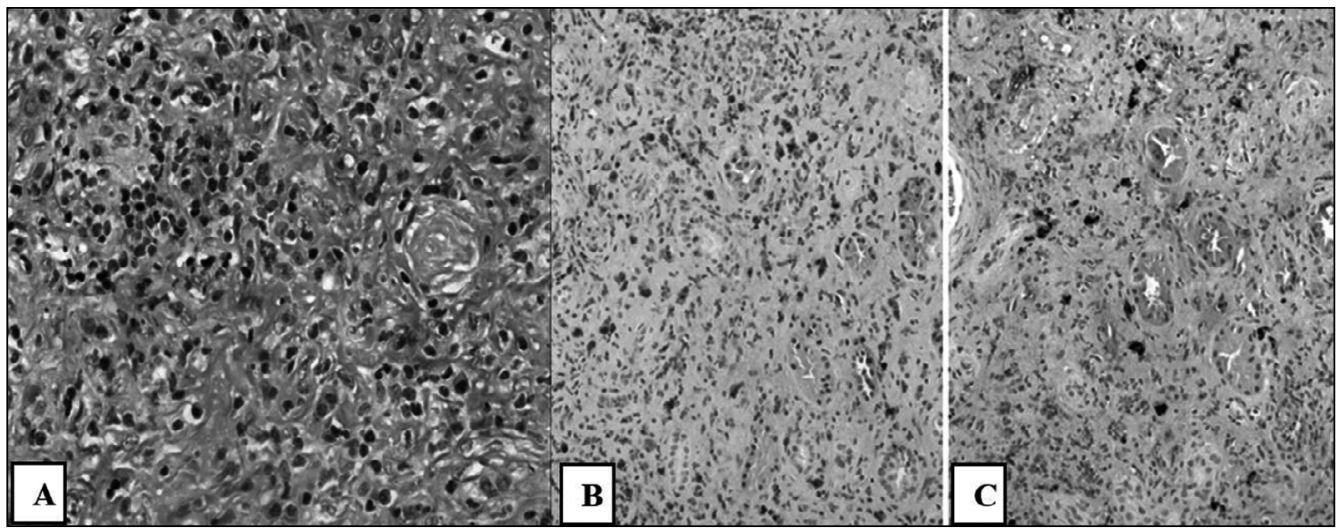

Figura 2. A: Zona del intersticio cortical que presenta un patrón de fibrosis con bandas colagenosas arremolinadas rodeando células inflamatorias (fibrosis "estoriforme"). Las células que componen el infiltrado inflamatorio intersticial corresponden a plasmocitos y linfocitos. (Tricrómico de Masson; 200x). B y C: Tinción inmunohistoquímica de zona cortical renal con anticuerpos contra lgG (B) e lgG4 (C). Se reconocen numerosos plasmocitos con reactividad positiva citoplasmática para IgG. Al observar la misma zona de infiltrado intersticial, se reconoce que la mayoría de los plasmocitos IgG positivos tienen también reactividad citoplasmática para IgG4. (B y C; 200x).

proteinuria. Nuevo estudio volvió a evidenciar hipocomplementemia y eosinofilia de igual magnitud, con serología reumatológica negativa, a excepción de factor reumatoídeo, positivo en 160 $\mathrm{U} / \mathrm{mL}$. Las crioglobulinas fueron negativas y en el recuento de inmunoglobulinas destacó una IgG de $2.898 \mathrm{mg} / \mathrm{dL}$ (normal < 1.600). Nuevamente el estudio bacteriológico, incluida la pesquisa de Mycobacterium, no mostró hallazgos. Responde favorablemente a tratamiento esteroidal y es dado de alta. Vuelve a consultar en dos ocasiones más por mismo cuadro, el 2014 y el 2015, momento en el cual se realiza biopsia renal, con el consentimiento del paciente, considerando el riesgo en su condi- ción de monorreno. En la biopsia se encontró una nefritis intersticial linfoplasmocitaria con extensa fibrosis de patrón estoriforme (arremolinada). La inmunohistoquímica confirmó positividad para IgG4 en los plasmocitos del infiltrado, con una relación IgG4/IgG de 63\% (Figura 2). La microscopía electrónica demostró depósitos electrón-densos en las membranas basales tubulares e intersticio. Se concluye nefritis intersticial relacionada a IgG4, más compromiso pulmonar muy probablemente asociado a la misma enfermedad. Se inicia tratamiento con prednisona $60 \mathrm{mg} /$ día, asociado a isoniazida para tratamiento de tuberculosis latente. Luego de 6 meses de tratamiento, se encuentra con 
$5 \mathrm{mg}$ de prednisona, creatinina de $1,45 \mathrm{mg} / \mathrm{dL}$, eosinófilos de $245 \mathrm{x} \mathrm{mm}^{3}$ y complemento C3/ C4 de $104 / 24 \mathrm{mg} / \mathrm{dL}$.

\section{Discusión}

La enfermedad renal relacionada con IgG4 presenta distintas manifestaciones. La nefritis tubulointersticial relacionada con IgG4 (NTIIgG4) es la afección más reconocida, sin embargo, se ha descrito también la glomerulonefritis y la arteritis de células plasmáticas ${ }^{2}$. Se presenta en el 15\% de los pacientes con ER-IgG4, siendo el $80 \%$ varones, con edad promedio de 65 años y asociándose en más del $80 \%$ a afección de otro órgano $^{2-4}$. La mayoría de los pacientes se presentan con disfunción renal crónica y en menor medida como un cuadro agudo de insuficiencia renal, proteinuria y hematuria microscópica. En ocasiones el estudio se inicia a través de alteraciones imagenológicas renales fortuitas o en el contexto de búsqueda de compromiso de otros órganos en pacientes con ER-IgG4. El edema de extremidades es infrecuente y cuando está, sugiere una glomerulonefritis asociada ${ }^{2,4}$. Los niveles de IgG4 elevados son el hallazgo serológico más representativo, pero pueden ser normales hasta en el 30\% de los casos $^{3}$. En el caso descrito, no se solicitaron dichos niveles, pues cuando se obtuvo el resultado de la biopsia con el inesperado diagnóstico, el paciente ya se encontraba en tratamiento esteroidal, situación que disminuye la sensibilidad del examen. El incremento en los niveles de IgG y la hipocomplementemia se presentan hasta en el $90 \%$ y $70 \%$ de los pacientes respectivamente, ésta última probablemente en relación a la presencia de depósitos de complemento en la membrana basal tubular. Otros hallazgos son el aumento en los niveles de IgE (70\%) y la eosinofilia $33-48 \%{ }^{5}$. El estudio de anticuerpos suele ser negativo, pero pueden existir títulos bajos de ANA (30\%) y factor reumatoídeo ${ }^{2,4,5}$. La proteinuria $(50 \%)$ suele ser menor a $1 \mathrm{~g} / 24$ horas y la microhematuria es un hallazgo frecuente. Un rasgo distintivo de la NTI-IgG 4 es la presencia de alteraciones imagenológicas que la diferencian de otros tipos de NTI. Las lesiones parenquimatosas por lo general son bilaterales, múltiples, afectan predominantemente la corteza y suelen invadir la cápsula renal. La TAC con contraste es la herramienta más útil, pero su uso requiere precaución en pacientes con función renal alterada ${ }^{4,5}$. La presencia de lesiones múltiples periféricas de baja densidad redondeadas o en cuña, es la expresión más frecuente (65\%). Otros patrones son el aumento difuso del tamaño renal (difícil de valorar en nuestro paciente considerando nefromegalia esperable por su condición de monorreno) y masa solitaria ${ }^{3-5}$. La ecografía puede mostrar nefromegalia e imágenes hipoecoicas. La resonancia magnética puede ser útil para detectar lesiones en fases tempranas en T2 y difusión ${ }^{5}$. La histopatología es el elemento diagnóstico clave. Los hallazgos principales en la microscopía de luz son la presencia de un denso infiltrado linfoplasmocitario y fibrosis en grado variable con patrón estoriforme. La flebitis obliterante, característica distintiva de la ER-IgG4, es raramente encontrada en el tejido renal. Otra característica es el aumento del número de eosinofilos y el compromiso de la capsula renal ${ }^{4}$. La presencia en la inmunohistoquímica de numerosas células plasmáticas IgG4 es esencial para el diagnóstico ${ }^{6}$. La presencia de más de 10 células plasmáticas IgG4 por campo mayor y la relación IgG4/IgG > 40 son muy características, pero pueden encontrarse en otros tipos de $\mathrm{NTI}^{6-8}$. Para realizar el diagnóstico de la NTI- IgG4 se requiere la combinación de elementos clínicos, serológicos, imagenológicos e histopatológicos ${ }^{3}$. En la actualidad existen 2 grupos de estudio con criterios diagnósticos bien definidos: el grupo americano de la Clínica Mayo y el de la Sociedad Japonesa de Nefrología $a^{3,4,9}$. Los primeros parecen ser más sensibles y pueden ser más útiles para la detección en fases tempranas de la enfermedad o casos atípicos. Los segundos son más estrictos y específicos ${ }^{10}$. La NTI-IgG4 requiere de tratamiento inmediato para suprimir la inflamación, evitar la fibrosis y preservar la función renal ${ }^{3,11}$. Los glucocorticoides son el tratamiento de primera línea. Reducen las "megalias", optimizan la función renal y disminuyen los niveles de IgG4. Un rasgo característico es la rápida respuesta a la terapia esteroidal, sin embargo, en los pacientes con NTI-IgG4 asociada a disfunción renal avanzada, la respuesta parece ser parcial ${ }^{4,11}$. El esquema más utilizado es el de la Sociedad Japonesa de Nefrología con prednisona a dosis de inducción de $0,6 \mathrm{mg} / \mathrm{kg} /$ día por $2-4$ semanas y posterior disminución progresiva hasta llegar a dosis de mantención de 5-10 mg día ${ }^{3-5}$. En casos de corticorresistencia o corticodependencia se han probado esquemas asociados con azatioprina o micofenolato con buena respuesta, aunque 
con evidencia limitada ${ }^{3}$. Existen algunos reportes con Rituximab con resultados prometedores ${ }^{3,12}$. La remisión espontánea es rara y las recaídas son frecuentes (20-30\%). Éstas pueden detectarse precozmente por niveles séricos elevados de IgG4 o la reaparición de hipocomplementemia ${ }^{4,5,12}$.

En relación al compromiso pulmonar de la ER-IgG4, se ha estimado que la afección parenquimatosa alcanza un $10 \%$, mientras que la pleural un $5 \%{ }^{13}$. En una serie de 21 pacientes con dicha afectación, un $43 \%$ presentó enfermedad extrapulmonar, simultánea o asincrónicamente ${ }^{14}$. A nivel parenquimatoso se describe principalmente pseudotumores y neumonía intersticial. En cuanto a la pleura; puede presentar engrosamiento y derra$\mathrm{me}^{14-16}$. Las manifestaciones clínicas varían desde lo asintomático hasta la tos, hemoptisis, disnea y dolor torácico ${ }^{13,17}$. Se han descrito cuatro patrones radiológicos de compromiso pulmonar: nódulos sólidos (el más frecuente), broncovascular (con engrosamiento de los haces broncovasculares y tabiques interlobulares), alveolo-intersticial (presencia de panal abeja, bronquiectasias, opacidad en vidrio esmerilado difusa) y opacidades en vidrio esmerilado "redondeadas"14,18. En muchos casos, el compromiso puede ser muy similar al de la sarcoidosis $^{19}$. Además del infiltrado linfoplasmocitario propio de la histología de la ER-IgG4, destaca la mayor frecuencia de flebitis obliterante. La respuesta a tratamiento esteroidal es buena y sólo algunos pacientes muestran lesiones radiológicas remanentes ${ }^{14}$.

\section{Referencias}

1. Pérez R, Espinoza L, Zea A. The great mimicker: IgG4-related disease. Clin Rheumatol 2013; 32 (7): 1267-73.

2. Pradhan D, Pattnaik N, Silowash R, Mohanty SK. IgG4-related kidney disease: A review. Pathol Res Pract 2015; 211 (10): 707-11.

3. Saeki T, Kawano M. IgG4-related kidney disease. Kidney Int 2014; 85 (2): 251-7.

4. Kim B, Kim JH, Byun JH, Kim HJ, Lee SS, Kim SY, et al. IgG4-related kidney disease: MRI findings with emphasis on the usefulness of diffusion-weighted imaging. Eur J Radiol 2014 Jul; 83 (7): 1057-62.

5. Baltar-Martín J, García Monteavaro C, Visus Fernández de Manzano T, Parejo García L. Afectación renal de las enfermedades relacionadas con IgG4. Nefro Plus 2015; 7 (1): 1-10.
6. Yoshita K, Kawano M, Mizushima I, Hara S, Ito Y, Imai N. Light-microscopic characteristics of IgG4-related tubulointerstitial nephritis: distinction from non-IgG4-related tubulointerstitial nephritis, Nephrol Dial Transplant 2012 Jul; 27 (7): 2755-61.

7. Jeong HJ, Shin SJ, Lim BJ. Overview of IgG4-related tubulointerstitial nephritis and its mimickers. J Pathol Transl Med 2016 Jan; 50 (1): 26-36.

8. Raissian Y, Nasr SH, Larsen CP, Colvin RB, Smyrk TC, Takahashi N, et al. Diagnosis of IgG4-related tubulointerstitial nephritis, J Am Soc Nephrol 2011 Jul; 22 (7): 1343-52.

9. Stone JH, Brito-Zerón P, Bosch X, Ramos-Casals M. Diagnostic approach to the complexity of IgG4-related disease. Mayo Clin Proc 2015 Jul; 90 (7): 927-39.

10. Khosroshahi A, Wallace ZS, Crowe JL, Akamizu T, Azumi A, Carruthers MN, et al. International consensus gidance statement on the management and treatment of IgG4-related disease. Arthritis Rheumatol 2015 Jul; 67 (7): 1688-99.

11. Saeki T, Kawano M, Mizushima I, Yamamoto M, Wada Y, Nakashima H, et al. The clinical course of patients with IgG4-related kidney disease. Kidney Int 2013 Oct; 84 (4): 826-33.

12. McMahon BA, Novick T, Scheel PJ, Bagnasco S, Atta MG. Rituximab for the treatment of IgG4-related tubulointerstitial nephritis: case report and review of the literature. Medicine (Baltimore) 2015 Aug; 94 (32): e1366.

13. Zen Y, Nakanuma Y. IgG4-related disease: a cross-sectional study of 114 cases. Am J Surg Pathol 2010; 34 (12): 1812-9.

14. Zen $\mathrm{Y}$, Inoue D, Kitao A, Onodera M, Abo H, Miyayama $\mathrm{S}$, et al. IgG4-related lung and pleural disease: a clinicopathologic study of 21 cases. Am J Surg Pathol 2009; 33 (12): 1886-93.

15. Taniguchi $T$, Ko $M$, Seko $S$, Nishida $O$, Inoue $F$, Kobayashi $\mathrm{H}$, et al. Interstitial pneumonia associated with autoinmune pancreatitis. Gut 2004; 53 (5): $770-1$.

16. Tsushima K, Tanabe T, Yamamoto H, Koizumi T, Kawa $\mathrm{S}$, Hamano $\mathrm{H}$, et al. Pulmonary involvement of autoimmune pancreatitis. Eur J Clin Invest 2009; 39 (8): 714-22.

17. Sun X, Liu H, Feng R, Peng M, Hou X, Wang P, et al. Biopsy-proven IgG4-related lung disease. BMC Pulm Med 2016 Jan 25; 16: 20.

18. Inoue D, Zen Y, Abo H, Gabata T, Demachi H, Kobayashi $\mathrm{T}$, et al. Immunoglobulin G4-related lung disease: CT findings with pathologic correlations. Radiology 2009; 251 (1): 260-70.

19. Tsushima K, Yokoyama T, Kawa S, Hamano H, Tanabe T, Koizumi T, et al. Elevated IgG4 levels in patients demonstrating sarcoidosis-like radiologic findings. Medicine (Baltimore) 2011; 90 (3): 194-200. 